\title{
Genital ulcer in 10-year-old girl: lipschütz ulcer
}

Keywords: genital ulcer, lipschütz ulcer, EBV, CMV, HIV, HBV, genital mucosa

\section{Clinical case}

A 10year old girl consulted by pain in the genital area for two days. The previous days, she has fever, cough and mucus. Pharyngeal pain. There are no changes in intestinal transit or trauma to the genital area.

Physical examination and complementary tests: ACP good bilateral ventilation without pathological noises, hyperemic pharynx without tonsillar exudates, not ulcer in the oral cavity. Laterocervical adenopathies movable, without pain. Normal abdominal examination. Ulcer of $1 \times 1 \mathrm{~cm}$ in right lower lip, around erythematous aloes. Hemogram and biochemistry with normal result. PCR $1.5 \mathrm{mg} / \mathrm{dl}$. Complete serology (lues, herpes simplex virus, EBV, CMV, HIV, HBV and $\mathrm{HCV}$, brucella) negative. Cultures urine and vaginal negatives. She received treatment with fusidic acid and surveillance. The ulcer disappeared at 2 weeks.

\section{Discussion}

The ulcer of Lipschütz is an acute ulceration that affect the genital mucosa in girls and adolescents. It evolution in a self-limited way. They are large ulcers, usually multiple and painful, with a grayish background. It may be accompanied by inguinal lymphadenopathy. Covered by a grayish membrane. The rest of the genitals are usually very oedematous and sensitive to the touch. Genital lesions appear and evolve in the context of a febrile syndrome, which is accompanied by asthenia, myalgia, odynophagia, lymphadenopathy or headache. The process is self-limited, and healing occurs spontaneously or after administration of broad-spectrum antibiotic therapy. Fever and symptoms disappear within a few days, and ulcers usually resolve within the first 2 weeks, although they can persist for one month. They heal without sequelae, scars and not recur.

The diagnosis of ulcer of Lipschütz is established by exclusion of other causes of acute genital ulceration, in particular venereal and other infections, and others less frequent such as physical or chemical trauma, Behçet's disease, lymphoma, pyoderma gangrenosum, disease inflammatory bowel disease, vulvular pemphigoid or recurrent idiopathic aphthosis. Histological examination is non-specific and does not allow differentiation of acute vulvar ulcer from its main differential diagnoses, although the histological study is not usually performed by the involution of the ulcer. ${ }^{1}$

The etiology is unknown, although an increasing number of cases associated with primoinfection by EBV have recently been
Volume 3 Issue I - 2017

Lydia Irles Díaz

Pediatrician, Clinic Center Polígono de Santa Ana, Spain

Correspondence: Lydia Irles Díaz, Pediatrician, Clinic Center Polígono de Santa Ana, Murcia, Spain, Email lydiairlesdiaz@hotmail.com

Received: October 13, 2017 | Published: November 02, 2017

described, even found by PCR in biopsies of lesions, ${ }^{2}$ although the pathophysiological mechanisms have not yet been clarified. It is also unknown why it affects almost exclusively women, since there is only one case documented in the specialized literature with affectation of the genitals of a male. ${ }^{3}$ Our patient presented a completely negative serology, thus discarding infectious causes. He had no cutaneous, digestive, joint, or ophthalmology symptomatology to guide another disease.

\section{Conclusion}

The ulcer of Lipschütz is an ulcer that appears in girls and adolescents. It is characterized by a painful lesion at the genital level, usually associated with fever, myalgias, headache and lymphadenopathy. The diagnosis is clinical although other causes must be ruled out. It has a complete spontaneous cure in about two weeks and does not need treatment except treatment for pain.

\section{Acknowledgement}

None.

\section{Conflict of interest}

Author declares that there is no conflict of interest.

\section{References}

1. Acute vulval ulcer or Lipschutz's disease. J Gynecol Obstet Biol Reprod. 31:684-686.

2. Pelletier F, Leblanc L, Estavoyer JM, et al. Ulcère de Lipschütz au cours d'une primo infection à virus Epstein-Barr. Ann Dermatol Venereol. 2002;129(6-7): 904-907.

3. Lawee D, Shafir MS. Solitary penile ulcer associated with infectious mononucleosis. Can Med Assoc J. 1983;129:146-147. 